\title{
AVALIAÇÃO DE MÉTODOS DE SELEÇÃO DE COMPETIDORES EM NÍVEL DE ÁRVORE INDIVIDUAL EM UMA FLORESTA ESTACIONAL SEMIDECIDUAL ${ }^{1}$
}

\author{
Liniker Fernandes da Silva ${ }^{2}$, Agostinho Lopes de Souza ${ }^{3}$, Carlos Pedro Boechat Soares ${ }^{3}$, Luiz Eduardo
} Sapori Gonçalves ${ }^{2}$ e Leonardo Pequeno Reis ${ }^{2}$

\begin{abstract}
RESUMO - Este estudo teve por objetivo avaliar a seleção de árvores competidoras com base em uma parcela circular com raio de $6 \mathrm{~m}$ e diâmetro das árvores superior ou igual a $5 \mathrm{~cm}$, no uso do fator de área basal igual a 1, de acordo com o método de Bitterlich, e na disputa por espaço e luz observados em campo. Para isso, fez-se a seleção dos fustes competidores ao redor de 24 árvores objeto, escolhidas em função de sua importância, no que concerne ao estoque de carbono e ao valor de seus produtos madeireiros e não madeireiros. Após as análises, observou-se, pelo teste $\mathrm{L} \& \mathrm{O}$, que ambos os métodos avaliados apresentaram baixa correlação em uma classe de diâmetro. Isso indica que a seleção dos competidores precisa ser mais estudada.
\end{abstract}

Palavras-chave: Floresta Estacional Semidecidual; Índices de competição; Fator de área basal.

\section{EVALUATING DIFFERENT METHODS TO SELECT COMPETITORS AT INDIVIDUAL TREE LEVEL IN A SEMIDECIDUOUS FOREST}

\begin{abstract}
The objective of this study was to evaluate the selection of competing trees based on a circular plot with a radius of 6 meters and diameter of trees equal or superior to $5 \mathrm{~cm}$; in the use of a basal area factor equal to 1, according to the Bitterlich's method; and based on the competition for space and light observed in the field. For this, there was the selection of stem competitors of about 24 trees, chosen due to their importance in relation to the carbon stock and value of their wood and non-wood products. After analysis, it was observed by the $L \& O$ test that both the evaluated methods showed low correlation in a diameter class. This indicates that the selection of competing trees needs to be more studied.
\end{abstract}

Keywords: Seasonal semideciduous forest; Competition indices; Basal area factor.

\section{INTRODUÇ̃̃̃O}

O crescimento é um processo em que ocorrem, entre outros, mudanças na forma e tamanho do tronco de uma árvore, com a consequente obtenção de novas camadas de lenho no material lenhoso já obtido (CAMPOS; LEITE, 2013).

A competição acontece quando duas árvores ou mais coabitam sobre uma mesma área de influência. Enquanto crescem, as plantas modificam o ambiente em que se encontram, reduzindo a oferta de recursos às plantas vizinhas (FORD; SORENSEN, 1992 apud
VANCLAY, 1995). Esses mesmos autores afirmaram ainda que o mecanismo primário de competição é a interação espacial, e a morte de plantas durante o processo de competição é efeito tardio da falta de recursos causada pela excessiva demanda de recursos. A competição tem efeitos significativos no crescimento e mortalidade (WYKOFF, 1900; QUICKE et al., 1994; HASENAUER; MONSERUD, 1996; STERBA etal., 2002). Assim, entende-se a importância da quantificação da competição para a avaliação do crescimento e mortalidade das árvores individuais, sobretudo em florestas nativas.

\footnotetext{
${ }^{1}$ Recebido em 13.04.2015 aceito para publicação em 29.10.2015.

${ }^{2}$ Universidade Federal de Viçosa, Programa de Pós-Graduação em Ciências Florestais, Viçosa, Minas Gerais - Brasil. E-mail: linikerfs@gmail.com>, <saporiluizeduardo@gmail.com>e $<$ leonardopequenoreis@gmail.com>.

${ }^{3}$ Universidade Federal de Viçosa, Departamento de Engenharia Florestal, Viçosa, Minas Gerais - Brasil. E-mail: <alsouzaal@gmail.com> e $<$ csoares@ufv.br>.
} 
Os modelos em nível de árvores individuais (MAI) consideram a árvore como unidade básica para a modelagem. São compostos de submodelos, entre eles os de mortalidade e crescimento da árvore, em altura e diâmetro. Várias informações, como os índices de competição (ZHAO et al., 2006), podem ser utilizadas como variáveis independentes nos diversos submodelos que compõem o MAI.

Os índices de competição têm por objetivo avaliar os efeitos das árvores vizinhas (competidoras) sobre a que se está analisando (árvore-objeto) (VANCLAY, 1995). Dessa forma, ele avalia o grau de supressão de cada árvore em relação às suas competidoras (MARTINS et al., 2011). Os índices de competição são classificados como dependentes da distância, independentes da distância e semi-independentes da distância (ALIG et al., 1984; STAGE; LEDERMANN, 2008).

Em estudo sobre índices de competição para um fragmento de Floresta Estacional Semidecidual em estágio médio de regeneração, os índices de competição semiindependentes da distância foram indicados para estudos de crescimento e mortalidade (CASTRO, 2014). O mesmo trabalho ainda indica um raio de $6 \mathrm{~m}$ para a seleção de indivíduos competidores.

A eficiência da modelagem em nível de árvores individuais pode ser maior quando se utilizam os índices de competição (LORIMER, 1983; CASTRO, 2014). Entretanto, a escolha dos competidores a serem considerados no cálculo dos índices de competição é, segundo Burton (1993), um problema ainda sem solução. Afinal, poucos trabalhos estudaram essa questão nas florestas nativas e sua aplicação para manejo sustentável de produtos florestais madeireiros e não madeireiros, sobretudo, para uso múltiplo (madeira, sementes, sequestro e estocagem de carbono).

Ciente da carência de estudos sobre a competição em florestas nativas e sabendo da importância deles na modelagem do crescimento e mortalidade, este estudo teve como objetivo fornecer mais informações a respeito da competição entre plantas, verificando-se a possibilidade do uso do fator de área basal de Bitterlich para a seleção de indivíduos competidores e avaliando se o raio de $6 \mathrm{~m}$ no entorno da árvore-objeto e a inclusão dos indivíduos de DAP superior a $5 \mathrm{~cm}$ são adequados para se trabalhar na modelagem do crescimento de Florestas Estacionais Semideciduais.

\section{MATERIAL E MÉTODOS}

\section{1. Área de estudo}

O local estudado foi uma Floresta Estacional Semidecidual Montana (VELOSO et al., 1991), denominada Mata da Silvicultura (coordenadas $42^{\circ} 52^{\prime} 30^{\prime \prime} \mathrm{W}$ e $20^{\circ} 46^{\prime} 10^{\prime \prime} \mathrm{S}$ ), localizada no campus da Universidade Federal de Viçosa, no Município de Viçosa, MG.

O clima da região é do tipo Cwb, temperado quente, segundo a classificação de Köppen, com verões quentes e chuvosos e invernos frios e secos. A temperatura média máxima é de $26^{\circ} \mathrm{C}$ e a média mínima, $14^{\circ} \mathrm{C}$. A precipitação média anual fica em torno de $1.300 \mathrm{~mm}$ (OLIVEIRA JUNIOR; DIAS, 2005).

Na região predominam classes de solo distintas: Latossolo Vermelho-Amarelo Álico (nos topos de morros e encostas); e Podzólico Vermelho-Amarelo Câmbico (nos terraços) (RESENDE et al., 1988).

O relevo da região de estudo é bastante acidentado, variando de forteondulado a montanhoso, tendo vales estreitos e úmidos e altitudes entre 600 e 970 m (CORREA, 1984). A área onde se localiza a Mata da Silvicultura tem altitude variando entre 680 e $760 \mathrm{~m}$.

A Mata da Silvicultura foi adquirida em 1936, e desde então a área se encontra em regeneração. Atualmente, o estágio sucessional pode ser interpretado como transição do médio para o avançado (MARISCAL FLORES, 1993; FIGUEIREDO, 2013).

Foram estudadas as espécies listadas a seguir, escolhidas em função do seu estoque de carbono na Mata da Silvicultura (valores em parênteses (FIGUEIREDO, 2015) na unidade $\mathrm{m}^{3}$.ha ${ }^{-1}$ ), bem como de seu potencial de aproveitamento para produtos madeireiros e não madeireiros:

- Anadenanthera peregrina (L.) Speng. (16,639).

- Pseudopiptadenia contorta (DC.) G. P. Lewis \& M.P. Lima $(7,425)$.

- Apuleia leiocarpa J. F. Macbr. (3,165).

- Copaifera langsdorffii Desf. (0,369).

- Dalbergia nigra Allemão ex Benth (0,657).

- Ocotea odorifera (Vell.) Rohwer $(1,175)$.

- Cariniana estrellenssis Kuntze (0,714). 


\section{- Cariniana legalis Kuntze.}

- Tapirira peckoltiana Engl. (0,332).

\subsection{Coleta dos dados}

Realizou-se o censo com mapeamento das árvores das espécies listadas com diâmetro mínimo de $20,0 \mathrm{~cm}$ em uma área com aproximadamente 7 ha. Identificouse a classificação botânica das árvores, mediu-se a circunferência a $1,3 \mathrm{~m}$ do solo (que foi posteriormente transformada para diâmetro), com o auxílio de uma fita, e estimou-se a altura total e do fuste com um hipsômetro Vertex.

As árvores encontradas no inventário com intensidade de amostragem $100 \%$ foram separadas em três classes de diâmetro, sendo elas:

- Comercial, com árvores de diâmetro superior ou igual a $50 \mathrm{~cm}$.

- Pré-comercial, com árvores de diâmetro mínimo de $35 \mathrm{~cm}$ e inferior a $50 \mathrm{~cm}$.

- Regeneração, com árvores de diâmetro mínimo de $20 \mathrm{~cm}$ e inferior a $35 \mathrm{~cm}$.

Para cada classe de diâmetro, foram escolhidas ao acaso oito árvores, totalizando 24 delas, que tiveram a competição no entorno avaliada. A seleção dos competidores no entorno da árvore em estudo foi realizada com as seguintes metodologias:

- Inclusão de todos os indivíduos com diâmetro mínimo de $5 \mathrm{~cm}$ em um raio de $6 \mathrm{~m}$ em torno da árvore objeto.

- Inclusão de competidores pelo método de Bitterlich com fator de área basal igual a 1 .

- Inclusão de indivíduos competidores com base na disputa por espaço e luz (nas espécies pioneiras e secundárias iniciais), observadas em campo, com base na copa das árvores.

O raio utilizado foi de $6 \mathrm{~m}$, pois gera valores do Índice de Competição com correlação significativa com a mortalidade em grande parte das espécies florestais na área estudada (CASTRO et al., 2014).

A observação da competição por espaço se deve ao fato de que o mecanismo primário da competição é a disputa por espaço (FORD; SORENSEN, 1992 apud VANCLAY, 1995). Tal afirmação é compreensível, tendo em vista que é em determinada área de influência que a planta obtém os recursos necessários para seu desenvolvimento.

A análise da disputa por luz, nas espécies secundárias iniciais, é compreensível, uma vez que não toleram o sombreamento em nenhuma fase de sua vida (BUDOWSKI, 1965). Já as secundárias tardias toleram o sombreamento em sua fase jovem, e assim as árvores desse grupo ecológico não tiveram a competição por luz avaliada.

Os competidores selecionados tiveram a circunferência à altura do peito (cap) medida com o auxílio de uma fita métrica e, posteriormente, a circunferência foi transformada em diâmetro. Além disso, as alturas comercial e total foram estimadas com o auxílio de um Vertex. Concomitantemente, avaliaram-se as seguintes variáveis categóricas: iluminação e qualidade de copa, qualidade do fuste e infestação de cipós. Os competidores foram identificados em nível de espécie, quando possível, com o auxílio de literatura especializada, consulta ao Herbário do Departamento de Biologia Vegetal da Universidade Federal de Viçosa e a botânicos especialistas. O sistema usado para a classificação florística foi o APG (2009).

\section{3. Índice de competição}

Foi utilizado o índice de competição dependente da distância que melhor explicou o crescimento e mortalidade na Mata da Silvicultura, em trabalho realizado por Castro (2014), ou seja:

Índice usado por Hegyi (1974)

$$
I C=\sum_{j=1}^{n j} \frac{\operatorname{dap}_{i}}{\operatorname{dap}_{j} \cdot L_{i j}}
$$

em que $d a p_{i}=$ diâmetro a 1,3 $\mathrm{m}$ de altura da árvoreobjeto $(\mathrm{cm}) ;$ dap $_{j}=$ diâmetro a 1,3 $\mathrm{m}$ de altura da árvore competidora (cm); e $L_{i j}=$ distância entre a árvore objeto e a árvore competidora $(\mathrm{m})$.

\subsection{Avaliação dos métodos de seleção dos competidores}

\subsubsection{Fator de área basal $(\mathrm{K})$ selecionado para cada classe de diâmetro}

Primeiramente, o objetivo foi encontrar, para cada classe de diâmetro, o fator de área basal, que teve maior semelhança com a metodologia de seleção dos fustes competidores baseada na disputa por espaço e luz.

Revista Árvore, Viçosa-MG, v.39, n.6, p.1119-1125, 2015 
Para isso, utilizou-se a seguinte fórmula:

$$
\sum_{c=j} d i f=\sum_{i=1}^{i=n} i c o-i d k
$$

em que dif é a diferença entre os métodos utilizados, ico é o método baseado na observação dos competidores por espaço e luz em campo, idk é o método baseado no fator de área basal, $j$ é a classe de diâmetro da árvore objeto e n é o n-ésimo fuste competidor selecionado.

Através de processo iterativo, encontrou-se para cada classe diamétrica o fator de área basal que minimizava a diferença entre as metodologias estudadas.

\subsubsection{Diferenças entre os métodos de seleção dos competidores}

Os três métodos de seleção de fustes competidores foram avaliados entre si, por meio do teste L\&O (LEITE; OLIVEIRA, 2002), dentro de cada classe de diâmetro avaliada neste trabalho. O teste em questão compara dois vetores combinando o teste $\mathrm{F}$ modificado por Graybill (1976) e um teste t no erro médio, além de uma análise na correlação.

\section{RESULTADOS}

\subsection{Avaliação dos métodos de seleção dos competidores}

\subsubsection{Fator de área basal $(\mathrm{K})$ selecionado para cada classe de diâmetro}

O comportamento das diferenças entre os métodos de seleção de fustes competidores, em que se identificaram os competidores por espaço e luz (apenas para árvores objeto pioneiras e secundárias iniciais) e os diversos fatores de área basal utilizados para seleção de competidores pode ser verificado na Figura 1. Com base nessas simulações, os fatores de área basal que mais se aproximaram da metodologia de seleção com base na competição por luz e espaço, para as classes de diâmetro de 20 a 35, de 35 a 50 e superior a $50 \mathrm{~cm}$, foram, respectivamente, 2,$48 ; 2,3$; e 3,4 (Tabela 1).

\subsubsection{Diferenças entre os métodos de seleção dos competidores}

Através do teste L\&O (Tabela 2), observou-se que o método de seleção de competidores com base no fator $\mathrm{k}$, obtido anteriormente, diferiu do método de seleção em campo apenas na classe com diâmetro

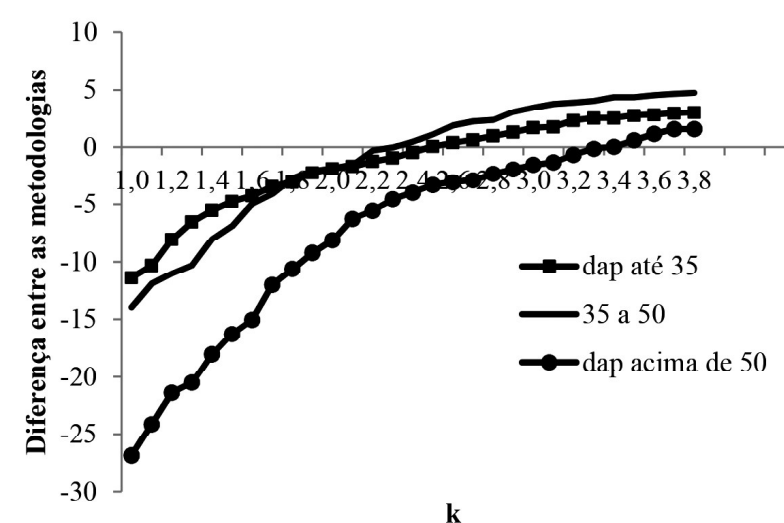

Figura 1 - Diferença do índice de competição de Hegyi no método de seleção de fustes competidores, em que foram selecionados os competidores por espaço e luz e método de seleção, tendo como base o fator de área basal $(\mathrm{K})$ para os diversos fatores.

Figure 1 -Difference of Hegyi competition index in the stem competitors selection method in which competitors were selected according to space and light and the selection method based on basal area factor $(K)$, for different factors.

Tabela 1 - Valores de fator de área basal (K) encontrados em cada classe de diâmetro na seleção de competidores, em uma Floresta Estacional Semidecidual montana.

Table 1 - Basal area factor values (K) found for each diameter class for selecting competitors in a semideciduous forest.

\begin{tabular}{ccc}
\hline Classe de diâmetro & $\mathrm{K}$ & Diferença \\
\hline $20 \leq$ dap $<35$ & 2,48 & $-0,0303$ \\
$35 \leq$ dap $<50$ & 2,3 & $-0,0295$ \\
$50 \leq$ dap & 3,4 & 0,0227 \\
\hline
\end{tabular}

entre 35 e $50 \mathrm{~cm}$. A seleção de todos os indivíduos encontrados dentro de um raio de $6 \mathrm{~m}$ com DAP igual ou superior a $5 \mathrm{~cm}$ diferiu do método de seleção em campo apenas na classe de diâmetro igual ou superior a $50 \mathrm{~cm}$.

\section{DISCUSSÃO}

O índice de competição de Hegyi pondera o peso da árvore competidora de acordo com a distância dela até a árvore objeto, diminuindo o efeito de árvores mais distantes no índice de competição. Tendo em mente que a interação espacial é o mecanismo de competição primário (FORD; SORENSEN, 1992 apud VANCLAY, 1995) e que o raio de $6 \mathrm{~m}$ não gerou médias do valor 
Tabela 2 - Teste L\&O comparando os valores do Índice de Competição de Hegyi usando diferentes métodos de seleção de fustes competidores em uma Floresta Estacional Semidecidual.

Table $2-L \& O$ test comparing the values of Hegyi competition index using different methods of stem competitors selection in a semideciduous forest.

\begin{tabular}{|c|c|c|c|c|c|c|}
\hline Classe de dap & Mét. 1 / Mét. 2 & $\mathrm{~F}\left(\mathrm{H}_{0}\right)$ & $\mathrm{t} \overline{\mathrm{e}}$ & $1-\overline{\mathrm{e}}$ & $r_{Y_{1}}, Y_{2}$ & Conclusão \\
\hline \multirow{2}{*}{$20<$ dap $<35$} & Ob. $/$ Raio $=6 \mathrm{~m}$ & $0,814^{\mathrm{ns}}$ & $2,074^{\mathrm{ns}}$ & $0,411<$ & 0,442 & Mét. $1=$ Mét. 2 \\
\hline & Obs. $/ \mathrm{k}$ & $0,484^{\mathrm{ns}}$ & $1,759^{\mathrm{ns}}$ & $0,535<$ & 0,698 & Mét. $1=$ Mét. 2 \\
\hline \multirow{2}{*}{$35<$ dap $<50$} & Ob. $/$ Raio $=6 \mathrm{~m}$ & $0,520^{\mathrm{ns}}$ & $1,627^{\mathrm{ns}}$ & $0,670<$ & 0,756 & Mét. $1=$ Mét. 2 \\
\hline & Obs. $/ \mathrm{k}$ & $1,370^{\mathrm{ns}}$ & $0,924^{\mathrm{ns}}$ & $0,868>$ & 0,388 & Mét. $1 \neq$ Mét. 2 \\
\hline \multirow{2}{*}{$\operatorname{dap}>50$} & Ob. $/$ Raio $=6 \mathrm{~m}$ & $0,824^{\mathrm{ns}}$ & $2,300^{\mathrm{ns}}$ & $0,251>$ & 0,223 & Mét. $1 \neq$ Mét. 2 \\
\hline & Obs. / k & $0,676^{\mathrm{ns}}$ & $1,905^{\mathrm{ns}}$ & $0,014<$ & 0,309 & Mét. $1=$ Mét. 2 \\
\hline
\end{tabular}

*Obs.: Mét. é o método de seleção dos indivíduos competidores; Ob. é o método de seleção dos indivíduos com base em observação de campo; Raio $=6 \mathrm{~m}$ é o método de seleção baseado na inclusão de indivíduos com dap $\geq 5 \mathrm{~cm}$ em um raio de $6 \mathrm{~m}$ em torno da árvore objeto; k é o método de seleção baseado no fator de área basal de Biterlich; è é a média dos erros entre os métodos avaliados; e $\mathrm{r}_{\mathrm{Y}_{1}}$, $\mathrm{Y}_{2}$ é a correlação entre os métodos avaliados.

do índice de competição distintas quando comparadas com a seleção de competidores por espaço e luz, pode-se assumir que a seleção de competidores com base no raio de $6 \mathrm{~m}$ e diâmetro mínimo de inclusão de $5 \mathrm{~cm}$ para o cálculo do índice de Hegyi é adequada, corroborando Castro (2014). Entretanto, o fato de a correlação não ter se mostrado satisfatória em alguns casos indica que a seleção de competidores para os cálculos de índices de competição gera valores muito dispersos, mesmo com média nula dos erros. Dessa forma, é necessária a condução de mais estudos sobre a seleção de competidores, como forma de gerar informações de maior precisão sobre a competição com o objetivo de aumentar a correlação entre os índices de competição e o crescimento ou mortalidade. Além disso, a avaliação da competição por luz é de fundamental importância, uma vez que ela afeta significativamente o comportamento das espécies arbóreas em uma floresta. Em trabalho realizado em uma Floresta Estacional Semidecidual, Castro et al. (2014) verificaram que pequenas árvores exigentes de luz tinham maior mortalidade. Espécies pioneiras e secundárias iniciais não toleram o sombreamento, enquanto as espécies secundárias tardias toleram o sombreamento em sua fase jovem (MARTINS, 2009).

Com isso, a recomendação do uso do raio de 6 $\mathrm{m}$ para estudos de crescimento em Florestas Estacionais Semideciduais (CASTRO et al., 2014) continua devido à falta de alternativas mais eficientes.

O uso do fator de área basal k na seleção de fustes competidores é alternativa viável nos estudos de crescimento em florestas inequiâneas, posto que, com o método, as médias do Índice de Hegyi não diferiram estatisticamente. Tal método tem como principal vantagem levar em consideração o tamanho e a distância entre a árvore objeto e a árvore competidora, o que o método de seleção baseado no raio de 6 m não faz. Assim, indica-se a necessidade de condução de estudos de crescimento que utilizam ambos os métodos, com o objetivo de compará-los.

\section{CONCLUSÕES}

Com base nos resultados deste estudo, pode-se concluir que:

- Os métodos de seleção dos competidores baseados no fator de área basal $\mathrm{k}$ e a medição de todas as árvores com diâmetro mínimo de $5 \mathrm{~cm}$ em raio de $6 \mathrm{~m}$ podem ser utilizados, entretanto é necessária a condução de estudos que visem melhorar a seleção dos competidores.

- Por ter sido utilizado com sucesso anteriormente e em razão da maior facilidade na seleção dos competidores, recomenda-se o uso do método que seleciona todos os fustes com diâmetro mínimo de 5 $\mathrm{cm}$ em raio de $6 \mathrm{~m}$ em torno da árvore-objeto.

\section{AGRADECIMENTOS}

Ao CNPq, pelo suporte financeiro a esta pesquisa.

\section{REFERENCIAS}

ALIG, R.J.; PARKS, P.J.; FARRAR Jr., R.M.; VASIEVIECH, J.M. Regional timber yield and cost information for the South: Modelings techniques. Washington, D.C.: USDA, 1984. 28p.

Revista Árvore, Viçosa-MG, v.39, n.6, p.1119-1125, 2015

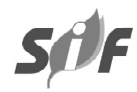


APG III. An update of the Angiosperm Phylogeny Group classification for the orders and families of flowering plants: APG III.

Botanical Journal of the Linnean Society, v. 161, p.105-121, 2009.

BUDOWSKI, G.N. Distribution of tropical American rain forest species in the light of succession processes. Turrialba, v.15, n.1, p.40-42, 1965.

CAMPOS, J.C.C.; LEITE, H.G. Mensuração florestal: perguntas e respostas. 4.ed. Viçosa, MG: UFV, 2013. 605p.

CASTRO, R.V.O.; SOARES, C.; LEITE, H.; SOUZA, A.; MARTINS, F.; NOGUEIRA, G.; OLIVEIRA, M.; SILVA, F. Competição em nível de árvore individual em uma Floresta Estacional Semidecidual. Silva Lusitana, v.22, n.1, p.43-66, 2014.

CORREA, G.F. Modelo de evolução e mineralogia da fração de argila de solos de planalto de Viçosa-mg. 1984. 187f. Dissertação (Mestrado em Solos e Nutrição de Plantas) - Universidade Federal de Viçosa, Viçosa-MG, 1984.

FIGUEIREDO, L.T.M.; SOARES, C.P.B.; SOUZA, A.L.; MARTINS, S.V. Alterações florísticas em uma Floresta Estacional Semidecidual, no município de Viçosa, MG, entre 1994 e 2008. Floresta, v.43, p.169-180, 2013.

FIGUEIREDO, L.T.M.; SOARES, C.P.B.; SOUZA, A.L.; LEITE, H.G.; SILVA, G.F. Dinâmica do estoque de carbono em fuste de árvores de uma Floresta Estacional

Semidecidual. CERNE, v.21, n.1, p.161-167, 2015.

HASENAUER, H.; MONSERUD, R.A. A crown ratio model for Austrian forests. Forest Ecology and Management, v.84, n.1, p.49-60, 1996.

HEGYI, F. A simulation model for managing jackpine stands. In: FRIES, J. (Ed.). Growth models for tree and stand simulation. Stockholm: Royal College of Forestry, 1974. p.74-90. (Note, 30).

Revista Árvore, Viçosa-MG, v.39, n.6, p.1119-1125, 2015
LEITE, H.G.; OLIVEIRA, F.H.T. Statistical procedure to test the identity of analytical methods. Communications in Soil Science and Plant Analysis, v.33, n.7/8, p.1105-1118, 2002.

LORIMER, C.G. Test of age-independent competition indices for individual trees in natural hardwood stands. Forest Ecology and Management, v.6, p.343-360, 1983.

MARISCAL FLORES, E.J. Potencial produtivo e alternativas de manejo sustentável de um fragmento de Mata Atlântica Secundária, Viçosa, Minas Gerais. 1993. 165f. Tese (Doutorado em Ciência Florestal) - Universidade Federal de Viçosa, Viçosa, MG, 1993.

MARTINS, S.V. (Org.). Ecologia de florestas tropicais do Brasil. Viçosa, MG: UFV, 2009. v.1.261p.

MARTINS, F.B.; SOARES, C.P.B.; LEITE, H.G.; SOUZA, A.L.; CASTRO, R.V. O. Índices de competição em árvores individuais de eucalipto. Pesquisa Agropecuária Brasileira, v.46, n.9, p.1089-1098, 2011.

OLIVEIRA JUNIOR, J.C.; DIAS, H.C.T.

Precipitação efetiva em fragmento secundário da Mata Atlântica. Revista Árvore, v.29, n.1, p.9$15,2005$.

RESENDE, M.; CURI, N.; SANTANA, D.P. Pedologia e fertilidade do solo: interações e interpretações. Brasília: Ministério da Agricultura, 1988.

STAGE, A.R.; LEDERMANN, T. Effects of competitor spacing in a new class of individualtree indices of competition: semi-distanceindependent indices computed for Bitterlich versus fixed-area plots. Canadian Journal of Forest Research, v.38, n.4, p.890-898, 2008.

STERBA, H.; BLAB, A.; KATZENSTEINER, K. Adapting an individual tree growth model for Norway Spruce (Picea abies L. Karst.) in pure and mixed species stands. Forest Ecology and Management, v.159, n.1, p.101-110, 2002. 
VANCLAY, J.K. Growth models for

tropical forests: a synthesis of models and methods. 1995. Disponível em: http://

espace.library.uq.edu.au/eserv/UQ:8366/

R048 fs pp.pdf. Acesso em: 25 mar 2013.

VELOSO, H.P.; RANGEL FILHO, A.L.R.; LIMA, J.C. Classificação de vegetação brasileira, adaptada a um sistema universal. Rio de Janeiro: IBGE, 1991.

QUICKE, H.E.; MEHLDAHL, R.S.; KUSH, J.S.

Basal area growth of individual trees: a model derived from a regional longleaf pine growth study. Forest Science, v.40, n.3, p.528-542, 1994.

WYKOFF, W.R. A basal area increment model for individual conifers in the northern rocku mountains. Forest Science, v.36, n.4, p.10771104, 1990.

ZHAO, D.; BORDERS, B.; WILSON, M.;

RATHBUN, S.L. Modeling neighborhood effects on the growth and survival of individual trees in a natural temperate species-rich forest.

Ecological Modelling, 2006. Disponível em: http://www.sciencedirect.com/science/article/pii/ S0304380006000585. Acesso em: 21 de jun de 2013. 
